\title{
Kinase Inhibitor Library Screening Identifies the Cancer Therapeutic Sorafenib and Structurally Similar Compounds as Strong Inhibitors of the Fungal Pathogen Histoplasma capsulatum
}

\author{
Charlotte Berkes ${ }^{1}{ }^{*}$, Jimmy Franco ${ }^{2} \mathbb{D}$, Maxx Lawson ${ }^{1}$, Katelynn Brann ${ }^{1}$, Jessica Mermelstein ${ }^{1}$, Daniel Laverty ${ }^{1,2}$ \\ and Allison Connors ${ }^{2}$ \\ 1 Department of Biology, Merrimack College, North Andover, MA 01845, USA; \\ lawsonm@merrimack.edu (M.L.); doironk@merrimack.edu (K.B.); mermelsteinje@gmail.com (J.M.); \\ lavertyd@merrimack.edu (D.L.) \\ 2 Department of Chemistry and Biochemistry, Merrimack College, North Andover, MA 01845, USA; \\ francoj@merrimack.edu (J.F.); connorsa@merrimack.edu (A.C.) \\ * Correspondence: berkesc@merrimack.edu
}

check for

updates

Citation: Berkes, C.; Franco, J.; Lawson, M.; Brann, K.; Mermelstein, J.; Laverty, D.; Connors, A. Kinase Inhibitor Library Screening Identifies the Cancer Therapeutic Sorafenib and Structurally Similar Compounds as Strong Inhibitors of the Fungal Pathogen Histoplasma capsulatum. Antibiotics 2021, 10, 1223. https:// doi.org/10.3390/antibiotics10101223

Academic Editor: Marc Maresca

Received: 25 August 2021

Accepted: 24 September 2021

Published: 8 October 2021

Publisher's Note: MDPI stays neutral with regard to jurisdictional claims in published maps and institutional affiliations.

Copyright: (c) 2021 by the authors. Licensee MDPI, Basel, Switzerland. This article is an open access article distributed under the terms and conditions of the Creative Commons Attribution (CC BY) license (https:/ / creativecommons.org/licenses/by/ $4.0 /)$.

\begin{abstract}
Histoplasma capsulatum is a dimorphic fungal pathogen endemic to the midwestern and southern United States. It causes mycoses ranging from subclinical respiratory infections to severe systemic disease, and is of particular concern for immunocompromised patients in endemic areas. Clinical management of histoplasmosis relies on protracted regimens of antifungal drugs whose effectiveness can be limited by toxicity. In this study, we hypothesize that conserved biochemical signaling pathways in the eukaryotic domain can be leveraged to repurpose kinase inhibitors as antifungal compounds. We conducted a screen of two kinase inhibitor libraries to identify compounds inhibiting the growth of Histoplasma capsulatum in the pathogenic yeast form. Our approach identified seven compounds with an elongated hydrophobic polyaromatic structure, five of which share a molecular motif including a urea unit linking a halogenated benzene ring and a para-substituted polyaromatic group. The top hits include the cancer therapeutic Sorafenib, which inhibits growth of Histoplasma in vitro and in a macrophage infection model with low host cell cytotoxicity. Our results reveal the possibility of repurposing Sorafenib or derivatives thereof as therapy for histoplasmosis, and suggest that repurposing of libraries developed for human cellular targets may be a fruitful source of antifungal discovery.
\end{abstract}

Keywords: Histoplasma; antifungal; Sorafenib; drug repurposing

\section{Introduction}

Treatment of systemic fungal infections presents a formidable challenge to the healthcare system. Recent advances in medicine have saved the lives of millions with diseases and conditions that were previously untreatable. However, these advances, including immunomodulatory therapies for cancer treatment, autoimmune disease, and organ transplantation have contributed to the increasing population of individuals with immunocompromised status who have a heightened susceptibility to fungal infection [1]. Invasive and/or systemic fungal infections carry a high cost in human and economic terms; in the United States alone in 2017, 4.5 billion USD was spent on the treatment of fungal infections requiring hospitalization [2]. Unfortunately, our arsenal of antifungal therapies has not expanded at a sufficient pace to meet the need.

The dimorphic fungal pathogens-Histoplasma, Blastomyces, Coccidioides and Paracoccidioides-are of particular clinical concern as these organisms infect and cause disease in both immunocompromised and healthy hosts. Histoplasma capsulatum is a dimorphic 
fungal pathogen and the etiologic agent of histoplasmosis, the most common fungal respiratory infection in the United States [3]. In endemic areas in the Midwestern U.S., the soil harbors a sporulating mold form of the organism that can be aerosolized and inhaled by mammalian hosts. Fungal spores germinate within the lungs and establish growth as budding yeasts, which are subsequently phagocytosed by alveolar macrophages, one of the primary host cells for $H$. capsulatum during infection. H. capsulatum yeasts replicate within the macrophage phagosome, ultimately leading to macrophage lysis [3]. Control of the infection in healthy hosts requires a robust $\mathrm{CD}^{+}$response; therefore, individuals with impaired cellular immunity are at risk of developing severe disseminated disease. While the clearance of Histoplasma in healthy individuals is poorly understood, there is evidence that in some individuals the infection can enter a latent state with the potential to re-emerge following a period of weakened immunity [4-7].

Currently, the primary antifungals available for the treatment of serious and lifethreatening fungal infections fall into just three mechanistic classes: the polyenes, azoles, and echinocandins. The polyene amphotericin B interacts with membrane ergosterol, thereby disrupting membrane integrity and acting in a fungicidal manner. Azole drugs function by interfering with ergosterol synthesis [8,9]. Treatment of acute and chronic pulmonary histoplasmosis as well as progressive disseminated disease relies on amphotericin B, sometimes followed by itraconazole [10]. The effectiveness of the deoxycholate formulation of amphotericin B is limited by renal and infusion-related toxicity, although toxicity has been partially mitigated by the development of liposomal formulations of amphotericin B [11,12]. The echinocandins inhibit synthesis of cell wall beta glucan and have been adopted for the treatment of disseminated candidiasis [13]. Unfortunately, Histoplasma and other dimorphic fungi harbor endogenous resistance to the echinocandins, limiting their effectiveness in the clinic [14,15].

A novel mechanistic class of antifungals has not been introduced to the clinic since the advent of the echinocandins two decades ago $[8,16]$. An expansion of the antifungal arsenal from which clinicians may draw from to treat patients with invasive fungal infections is long overdue. The barriers interfering with development of antifungal agents to treat invasive fungal infections are both scientific and economic/regulatory. Due in large part to the eukaryotic nature of fungi, the universe of structurally unique targets to be exploited for antifungal therapy is much smaller than for that of prokaryotic pathogens. Intracellular fungal pathogens such as Cryptococcus and Histoplasma present additional challenges in antifungal delivery due to the protected nature of their respective host cell niches. Antifungal compounds that are effective in vitro are unfortunately more likely than not to fail to clear regulatory hurdles. Drug repurposing has been an effective approach for a number of infectious disease targets [17]; the significant challenges of histoplasmosis treatment prompts consideration of a repurposing approach for Histoplasma.

In eukaryotic systems, protein phosphorylation switches play a central role in signaling pathways regulating nearly all cellular processes including proliferation, differentiation, and response to stress. Kinase inhibition has been a highly effective strategy for the development of anti-cancer therapies, and development of kinase inhibitor libraries optimized for drug-like qualities has supported and accelerated research in this area in recent years [18-20]. In the model yeast Saccharomyces cerevisiae, MAP kinase cascades have been shown to regulate response to osmotic stress, mating, cell wall integrity and ascospore formation [21-25]. Evidence from knockout studies in the model fungal pathogens Candida albicans [26-28] and Cryptococcus neoformans [29-31] have highlighted the roles of kinases in virulence. The conservation of protein kinase signaling throughout the eukaryotic domain suggests that these pathways may be pharmacologically modulated as a strategy to combat fungal and protozoan infections [32]. These strategies may employ kinase inhibitors to directly interfere with cellular machinery required for fungal virulence and/or viability, or may be used to amplify their effectiveness by interfering with antifungal resistance mechanisms [33]. 
In the current study, we screened two kinase inhibitor libraries to identify compounds inhibiting the growth of Histoplasma capsulatum in the pathogenic yeast form. From these libraries, we identified a set of seven compounds containing an elongated hydrophobic polyaromatic structural motif that inhibit growth of Histoplasma capsulatum in the low micromolar/nanomolar range. Five of the seven compounds contain highly similar molecular structures containing the chemical motif of a urea unit linking a halogenated benzene ring and a para-substituted polyaromatic group. One of these compounds, Sorafenib, is an FDA-approved compound currently used for treatment of renal small cell carcinomas. Sorafenib inhibits growth of Histoplasma both in vitro and in a macrophage infection model with low host cell cytotoxicity. Our work highlights the potential to repurpose Sorafenib as therapy for histoplasmosis, and identifies a molecular motif for further development.

\section{Results}

\subsection{Kinase Inhibitor Library Screening Identifies Anti-Histoplasma Compounds}

Two kinase inhibitor libraries were screened in an effort to identify novel molecules inhibiting Histoplasma growth. The GlaxoSmithKline Published Kinase Inhibitor Set (GSK PKIS) is a collection of 367 small molecule ATP-competitive kinase inhibitors optimized for drug-like physicochemical properties and developed for diverse kinase targets [34,35]. Histoplasma capsulatum WU15 (Hc WU15), a uracil auxotrophic strain derived from the clinical G217B isolate, was grown in microtiter plates in combination with GSK PKIS library compounds at a final concentration of $5 \mu \mathrm{M}$ and growth was monitored over the course of four days by optical density determination as previously described [36,37]. Three compounds, GW778894X, GW770249A, and GW795486X, inhibited growth of Hc WU15 by greater than 50\% relative to amphotericin B, with GW778894X and GW770249A displaying the most severe growth inhibition (Figure 1A). Given the evidence supporting a central role of fungal MAP kinase homologs in stress response and virulence, we also screened a commercially available library of 61 cell permeable inhibitors targeting MEK, Raf, p38, JNK, ERK and other MAP kinases. The MAP kinase library yielded three compounds meeting criteria for $50 \%$ growth inhibition over a four day time course as monitored by optical density: GW5074, Sorafenib, and the tosylate salt formulation of Sorafenib (Figure 1B).

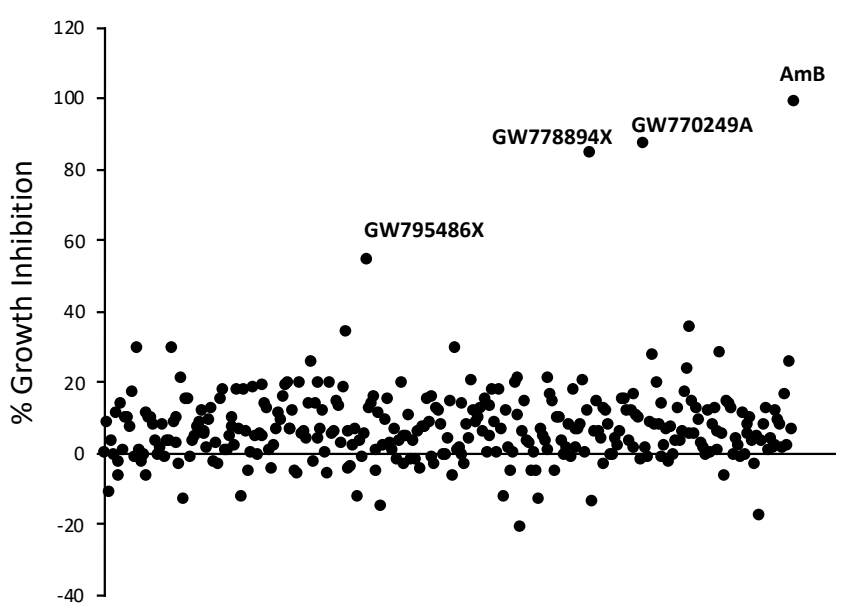

(A)

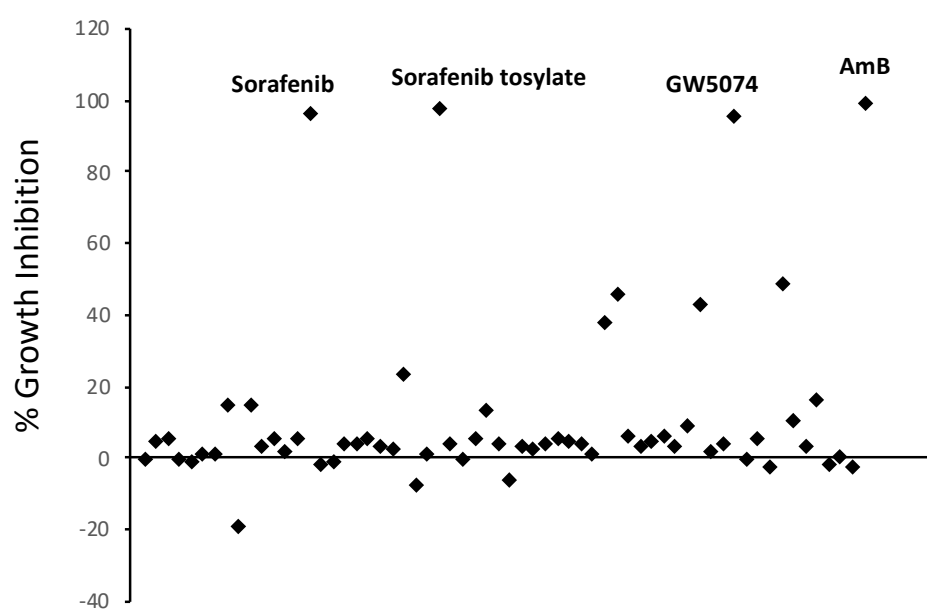

(B)

Figure 1. Graphical representation of primary screen results of the Published Kinase Inhibitor Set (A) and the SelleckChem MAPK Inhibitor Library (B) against Histoplasma capsulatum. Each individual compound was tested, in duplicate, at a final concentration of $5 \mu \mathrm{M}$. Log phase Hc WU15 yeasts were seeded in 96 well plates at a density of $1 \times 10^{6}$ yeasts $/ \mathrm{mL}$ in $\mathrm{HMM}$ / uracil. Compounds were diluted in DMSO and added to each well at a final DMSO concentration of $0.5 \%$. Plates were incubated at $37^{\circ} \mathrm{C}$ and $5 \% \mathrm{CO}_{2}$ with twice-daily shaking to improve aeration. $\mathrm{OD}_{595}$ readings were taken on day 0 and day 4 . The results for each compound are shown as average percent growth inhibition over the four day time period relative to the DMSO control. All compounds showing greater than 50\% growth inhibition at day 4 are identified. 
The chemical structures of top kinase inhibitor library hits are shown in Figure 2, highlighting the commonality of an elongated hydrophobic polyaromatic structural motif. Five of the six molecules identified in our screening efforts adhere to a structural motif containing a urea unit linking a halogenated benzene ring and a para-substituted polyaromatic group. The halogenated benzene portion of the molecule consists of either a F, Cl, or trifluoromethyl group, which are nonclassical bioisoteres of each other [38]. Additionally, most compounds exhibited favorable calculated $\log P$ values and satisfied Lipinski's rule of five for drug-likeness [39,40].

(A)<smiles>CNC(=O)c1cc(Oc2ccc(NC(=O)Nc3ccc(Cl)c(F)c3)cc2)ccn1</smiles><smiles>CNC(=O)c1cc(Oc2ccc(NC(=O)Nc3ccc(Cl)c(C(F)(F)F)c3)cc2)ccn1</smiles><smiles>Nc1ccc(Oc2ccc(NC(=O)Nc3ccc(Cl)c(C(F)(F)F)c3)cc2)cc1</smiles>

SC-1

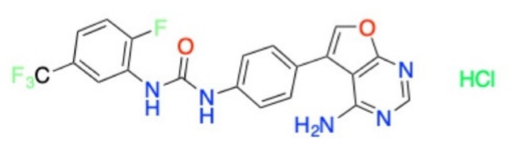

GW770249A

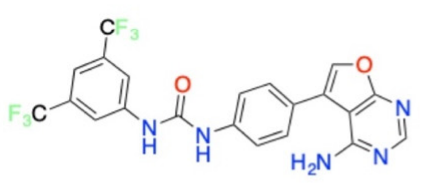

GW795486X<smiles></smiles>

GW 5074

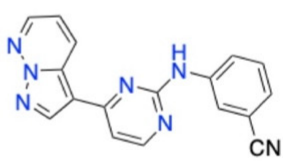

GW778894X

(B)

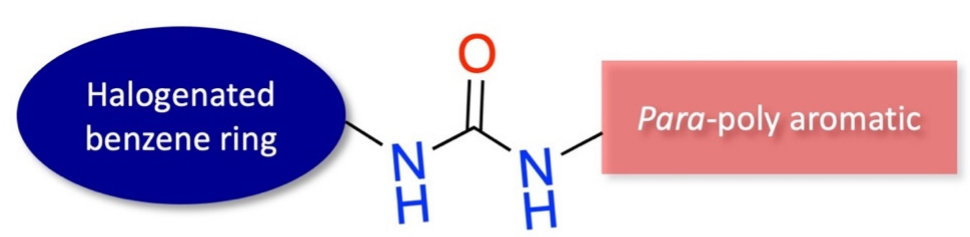

Figure 2. Chemical structures of compounds strongly inhibiting H. capsulatum growth at MIC $<2 \mu \mathrm{M}$. Sorafenib, Sorafenib tosylate, and GW5074 were identified in the SelleckChem library screen. SC -1 was included due to its structural similarity to Sorafenib. GW778894X, GW770249A, and GW795486X were identified in the PKIS library. (A) All seven of the compounds have an elongated hydrophobic polyaromatic structural motif. Five of the seven compounds contain highly similar molecular structures containing the chemical motif of a urea unit linking a halogenated benzene ring and a para substituted poly aromatic group, represented schematically in (B). 


\subsection{Characterization of Sorafenib and SC-1 Antifungal Properties}

Sorafenib is an FDA approved multi-kinase inhibitor used for the treatment of renal cell carcinoma, hepatocellular carcinoma, and metastatic differentiated thyroid cancer. Sorafenib and its close structural derivative, SC -1 (devoid of Raf inhibitory activity), have both been shown to have anti-tumor and anti-fibrotic effects in a variety of cancer cell lines and in vivo models [41-43]. We hypothesized that SC -1 would also have a strong inhibitory effect on the growth of $H$. capsulatum based on the fact that it shares the same diarylurea structural motif as many of our kinase library screen hits. MIC and $\mathrm{IC}_{50}$ values for Sorafenib tosylate and SC -1 were determined by exposing Hc WU15 to twofold serial dilutions of Sorafenib tosylate or SC -1 using protocols established previously for $H$. capsulaum $\mathrm{G} 217 \mathrm{~B}$ yeast form cultures [36,37]. Multiple dose response experiments determined the $\mathrm{IC}_{50}$ of Sorafenib tosylate and $\mathrm{SC}-1$ are $0.69 \mu \mathrm{M}$ and $0.15 \mu \mathrm{M}$, respectively (Figure 3A,B). The MIC values for Sorafenib tosylate and SC -1 are $1.0 \mu \mathrm{M}$ and $0.25 \mu \mathrm{M}$, respectively (Figure 3 and Table 1).

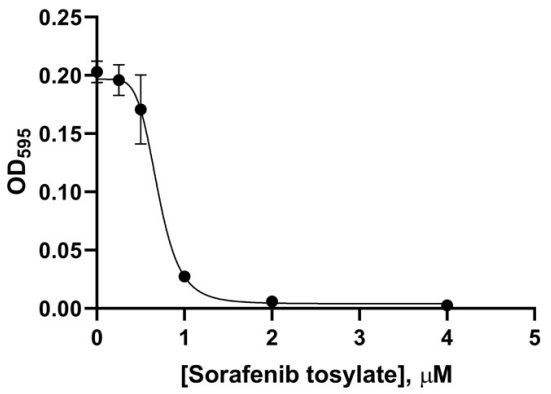

(A)

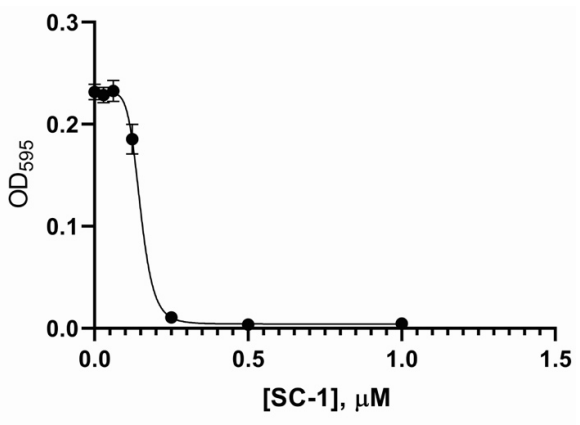

(B)

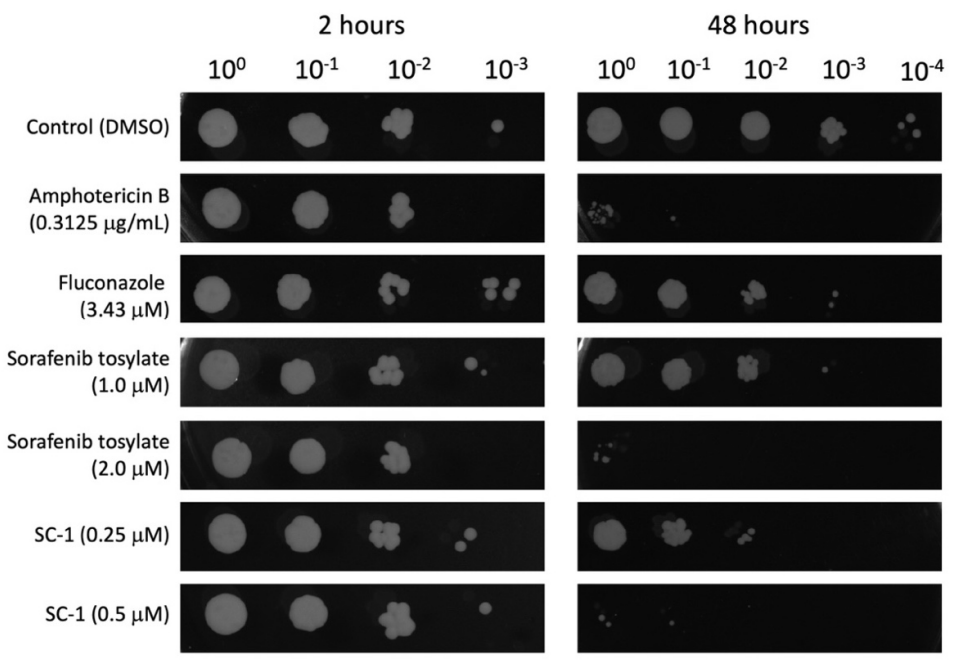

(C)

Figure 3. Sorafenib and SC -1 are fungistatic to $H$. capsulatum yeasts. Hc WU15 yeasts were seeded in 96 well plates at a density of $4 \times 10^{6}$ yeasts $/ \mathrm{mL}$ in HMM. Sorafenib tosylate (a) or SC-1 (b) were diluted in DMSO and added to each well at a final DMSO concentration of $0.5 \%$ with a two-fold dilution series of each compound. Growth of yeasts was monitored by measuring absorbance at $595 \mathrm{~nm}$. $\mathrm{IC}_{50}$ values were calculated using nonlinear regression of the data at the 4 day time point. (c) Fungistasis was demonstrated by plating serial dilutions of yeasts onto solid media at 2 and $48 \mathrm{~h}$ following addition of compounds to measure the number of viable CFU. The images shown are representative of four independent experiments. 
Table 1. Minimum inhibitory concentration (MIC) for top library screen hits against Histoplasma capsulatum WU15 and WU8, Cryptococcus neoformans H99, and Candida albicans SC5314.

\begin{tabular}{|c|c|c|c|c|c|c|c|}
\hline Library & Compound & Hc WU15 & Hc WU8 & C. neoformans & C. albicans & $\log P$ & $\begin{array}{l}\text { Satisfies Lipinski's } \\
\text { Rule of } 5 ?\end{array}$ \\
\hline \multirow{3}{*}{ PKIS } & GW770249A & 0.5 & 2.0 & $>50$ & $>50$ & 3.65 & Yes \\
\hline & GW7788994X & 0.25 & 1.0 & $>50$ & $>50$ & 1.21 & Yes \\
\hline & GW795486X & 2.0 & 4.0 & $>50$ & $>50$ & 4.41 & Yes \\
\hline & GW5074 & 2.0 & 4.0 & $>50$ & $>50$ & 5.31 & Partially \\
\hline \multirow[t]{3}{*}{ SelleckChem } & $\begin{array}{c}\text { Sorafenib } \\
\text { tosylate }\end{array}$ & 1.0 & 2.0 & 25 & $>50$ & 3.76 & Yes \\
\hline & Sorafenib & 1.0 & 2.0 & 25 & $>50$ & 3.8 & Yes \\
\hline & SC-1 & 0.25 & 1.0 & 12.5 & $>50$ & 5.56 & Partially \\
\hline
\end{tabular}

To determine the spectrum of antifungal activity, we tested all compounds identified in the library screening experiments, as well as SC -1 , against additional clinically relevant and model fungi (Table 1). None of the compounds interfered with growth of Saccharomyces cerevisiae or Candida albicans up to the highest concentration tested (50 $\mu \mathrm{M})$. However, Sorafenib and SC-1 showed weak activity against Cryptococcus neoformans (Table 1). H. capsulatum G186AR is a "rough" isolate that is phylogenetically distinct from G217B. We tested the compounds against a uracil auxotrophic strain of $H$. capsulatum G186AR (Hc WU8), and found similar MIC values as for WU15.

To determine whether Sorafenib and SC -1 function in a fungistatic or fungicidal manner, we cultured Hc WU15 in media containing these compounds for $2 \mathrm{~h}$ and $48 \mathrm{~h}$, then plated cultures on drug-free solid media. Additional wells contained amphotericin B or fluconazole, which inhibit $H$. capsulatum via fungicidal and fungistatic mechanisms, respectively. We used drug concentrations at the previously published MIC for amphotericin B $(0.3125 \mu \mathrm{g} / \mathrm{mL})$ and fluconazole $(3.43 \mu \mathrm{M})$; we tested Sorafenib tosylate and SC -1 at their respective MICs $(1.0 \mu \mathrm{M}$ and $0.25 \mu \mathrm{M})$ and at double the MIC values $2.0 \mu \mathrm{M}$ and $0.5 \mu \mathrm{M}$, respectively). Interestingly, both Sorafenib and $\mathrm{SC}-1$ act in a fungistatic manner at the MIC concentration, whereas at $2 \times$ MIC the antifungal activity is fungicidal (Figure $3 C$ ).

To determine whether Sorafenib and/or SC -1 act synergistically with traditional antifungals, we performed checkerboard assays in which Sorafenib or SC -1 was added to $\mathrm{Hc}$ WU15 cultures in combination with fluconazole at a range of concentrations. In these experiments, we failed to observe synergy between Sorafenib or SC -1 and fluconazole (Supplementary File).

\subsection{Effectiveness of Sorafenib and SC-1 against Intracellular H. capsulatum}

During an infection, H. capsulatum cells reside within the phagosomal compartment of alveolar macrophages where they persist and evade immune clearance. Therefore, anti-Histoplasma compounds must be able to target intraphagosomal yeasts in order to be effective. H. capsulatum replication within the macrophage phagosome culminates in macrophage lysis, and the ability of yeasts to stimulate lysis is required for virulence in vivo [44]. To further explore the potential to repurpose Sorafenib for antifungal therapy, we tested the ability of Sorafenib to inhibit lysis of host macrophages. Murine bone marrow-derived macrophages (BMDM) were infected with $H$. capsulatum at a multiplicity of infection of 5. After co-incubation of yeasts with BMDM for $2 \mathrm{~h}$, extracellular yeasts were removed by washing with media and cultures were treated with DMSO or Sorafenib tosylate at a range of concentrations. The extent of macrophage lysis was determined by monitoring release of lactate dehydrogenase (LDH). At $48 \mathrm{~h}$ following infection, visual inspection of BMDM cultures treated with DMSO showed robust yeast growth culminating in BMDM lysis, as indicated by release of LDH into the culture media. Samples treated with Sorafenib tosylate showed a dose-dependent decrease in BMDM lysis up to $6.25 \mu \mathrm{M}$ (Figure 4). Consistent with previously demonstrated cytotoxicity in mammalian cells at concentrations greater than 5-10 $\mu \mathrm{M}$, wells treated with higher Sorafenib tosylate concen- 
trations showed increased lysis (Figure 4). Taken together, these results are consistent with effective delivery of Sorafenib to the macrophage phagosome.

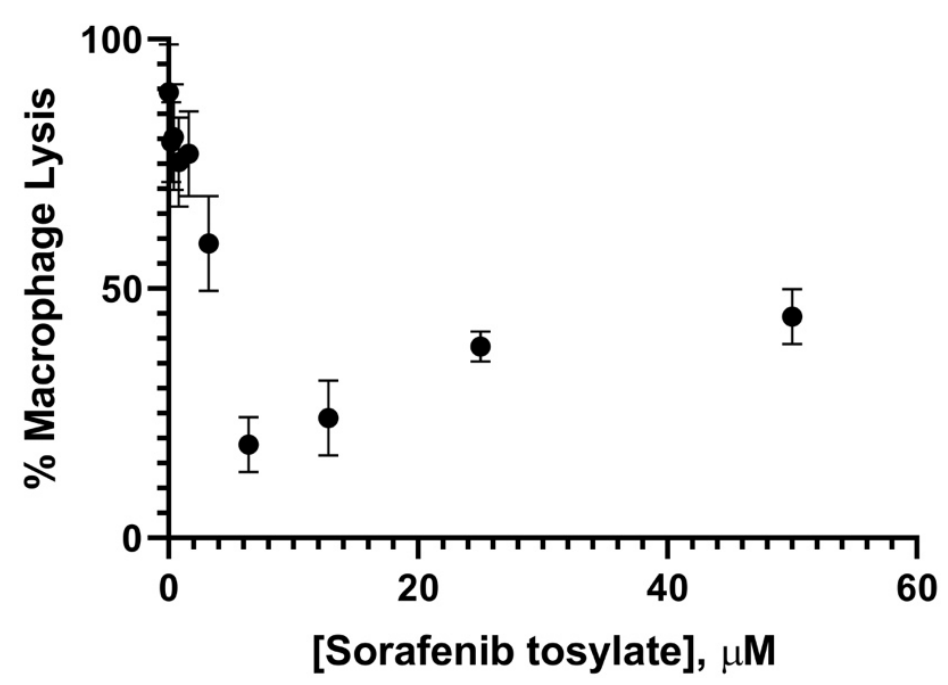

Figure 4. Sorafenib inhibits growth of intracellular H. capsulatum and macrophage lysis. Murine BMDM were seeded in 24 well tissue culture treated wells and infected with Hc WU15 at an MOI of 5. After two hours, extracellular yeasts were removed by washing and media containing Sorafenibtosylate or DMSO only was added, with each condition in triplicate. Following $48 \mathrm{~h}$ incubation at $37{ }^{\circ} \mathrm{C}$ and $5 \% \mathrm{CO}_{2}$, macrophage lysis was monitored by CytoTox LDH release assay. \% lysis was calculated relative to a detergent-treated uninfected control. Data shown are representative of three independent experiments.

\section{Discussion}

The specific aim of this study was to identify kinase inhibitors with anti-Histoplasma activity, an approach that has previously been successful for both fungal and protozoan pathogens [45-48]. Our approach is based on a repurposing strategy in which conservation of kinase mediated signaling pathways in all eukaryotes may be leveraged to identify compounds within libraries optimized for drug-like qualities. Here, we present an adaptation of antifungal screening methods developed for the pathogenic yeast form of Histoplasma capsulatum $[36,37]$ that utilizes an avirulent uracil auxotrophic strain cultured in media supplemented with uracil. We use this method to screen two kinase inhibitor libraries for compounds inhibiting Histoplasma growth. have an elongated polyaromatic motif and calculated $\log$ P values of 5.6 or less, which is generally considered drug-like and beneficial for absorption and permeability $[39,40]$. Similar to the recently identified aminothiazole derivatives demonstrating activity against Histoplasma capsulatum, the compounds identified here contain a central amide with cyclic hydrophobic groups on either side of the amide [36]. They also have predominantly elongated motifs, similarly to previously identified aminothiazole derivatives, which displayed greater activity when substituted at the 2,5 position of the aminothiazole ring, when compared to the 3 and 4 position [49]. Significantly, our approach identified the cancer therapeutic Sorafenib as a robust inhibitor of Histoplasma capsulatum growth.

Sorafenib and several of the compounds identified in our screen are diarylureas, which has served as an important molecular scaffold in anticancer drug development [50,51]. A growing body of work shows a strong potential for the use of the diarylurea scaffold in the development of antiviral, antiparasite, antibacterial and antifungal compounds [52-54]. Sorafenib in particular is reported to possess activity against drug-resistant Staphylococcus aureus and to interfere with replication of New World alphaviruses through a mechanism that is independent of host cell kinase inhibition [55]. Others have shown that other diarylureas possess antifungal activity [56], but ours is the first report that Sorafenib and SC-1 possess antifungal activity. 
More generally, our work adds to a growing body of literature that has demonstrated the dual nature of anti-cancer or immunomodulatory compounds as antifungals [47,52]. The topoisomerase I-targeting compounds camptothecin and nitidine are active against Cryptococcus and Saccharomyces, and an exploration of structure-activity relationships for these drugs has identified chemical moieties associated with higher fungal selectivity [57]. The immunosuppressant rapamycin is also a potent antifungal agent whose activity is mediated via association with peptidylprolyl isomerase FKBP12, a target broadly conserved in both yeast and mammalian cells that forms a toxic complex with the TOR1 and TOR2 proteins in yeast or mTOR1 and mTOR2 proteins in mammals [58]. Wortmannin, a powerful immunosuppressant that acts via inhibition of PI-3 kinase in mammalian cells, exhibits antifungal activity that may be mediated by PI-4 and additional targets in yeast cells [59].

Sorafenib (BAY -43-9006 Nexavar, Bayer Pharmaceuticals Corp. and Onyx Pharmaceuticals Inc.) is a chemotherapeutic indicated for the treatment of hepatocellular carcinoma (HCC), renal cell carcinoma, and metastatic differentiated thyroid cancer. It is an orally active multi-kinase inhibitor that acts as a cytotoxic chemotherapeutic, inhibiting tumor cell proliferation and inducing apoptotic cell death via inhibition of VEGF and PDGF receptors as well as the Raf/MEK/ERK pathway [60]. SC-1 is a Sorafenib derivative lacking the functional amide group that confers Raf kinase inhibition activity upon Sorafenib. SC -1 is, however, a potent apoptosis inducer, functioning via a mechanism that includes inhibition of pSTAT3. The cytotoxicities of Sorafenib and its derivative, SC -1 , on HepG2 and other human cell lines have been documented previously [41-43,61,62].

In yeasts, the high osmolarity-glycerol (HOG) pathway is a MAP kinase-mediated cascade controlling critical aspects of stress response including osmoregulation and cell wall integrity (reviewed in [63]). This pathway has been demonstrated to be a feasible target for antifungal development in a classic study of the antifungal compound ambruticin, which demonstrates its action via inhibition of the high osmolarity-glycerol pathway in the yeast Hansenula anomala [64]. Our results do not directly show that Sorafenib and/or SC-1 mediate growth inhibition in Histoplasma by mechanisms parallel to those demonstrated in mammalian cells. However, it is interesting to note that one of the human targets of Sorafenib, p38, displays $52 \%$ identity and $69 \%$ similarity at the amino acid level with its H. capsulatum homolog, Hog1. Further studies are needed to explore the mechanism by which Sorafenib and SC -1 mediate fungistasis in H. capsulatum.

\section{Conclusions}

The results of this study show that Sorafenib, $\mathrm{SC}-1$, and structurally similar diarylureas inhibit the growth of H. capsulatum in the low micromolar range. Our study expands upon a previous literature demonstrating the potential use of antineoplastic drugs as antifungal agents, and is significant in that it specifically identifies the potential to repurpose Sorafenib and/or its derivatives as antifungal therapies. Furthermore, our findings support the concept of kinase inhibitor repurposing for fungal targets. Future work will establish a structure-activity relationship and optimize the effectiveness of Sorafenib derivatives for inhibition of Histoplasma.

\section{Materials and Methods}

\subsection{Fungal Strains and Culture}

H. caspsulatum WU15 (G217B ura5 $\Delta$ ) and WU8 (G186AR ura5 $\Delta$ ) have been described previously [65]. In uracil-replete media, G217B and WU15 display comparable growth rates and kinetics of macrophage lysis [44,66]. H. caspsulatum WU15 and WU8 cultures were maintained in yeast phase by culturing in liquid Histoplasma macrophage media (HMM) supplemented with $200 \mu \mathrm{g} / \mathrm{mL}$ uracil (Sigma-Aldrich, St. Louis, MO, USA) at $37^{\circ} \mathrm{C}$ and $5 \% \mathrm{CO}_{2}$ with constant agitation. Candida albicans SC5314 (ATCC MYA-2876), Saccharomyces cerevisiae BY4741 (ATCC 201388), and Cryptococcus neoformans H99 (ATCC 208821) were maintained in liquid or solid YPD media at $30^{\circ} \mathrm{C}$. 


\subsection{Chemical Library Screening}

The GlaxoSmithKline Published Kinase Inhibitor Set (PKIS) was supplied by the University of North Carolina Structural Genomics Consortium under an open access Material Transfer and Trust Agreement. The PKIS library and the MAP kinase inhibitor library L3400 (SelleckChem, Houston, TX, USA) were provided as $1 \mathrm{mM}$ stocks in DMSO. Immediately prior to experiments with $H$. capsulatum, $2 \times$ working stocks of compounds were prepared in HMM media. H. capsulatum cultures were prepared as previously described $[36,37]$. Briefly, overnight cultures in log phase were counted using a hemacytometer. Yeasts were seeded at $1.0 \times 10^{6}$ yeasts $/ \mathrm{mL}$ in a final volume of $50 \mu \mathrm{L}$, to which $50 \mu \mathrm{L}$ of the kinase inhibitor working solutions were added such that the final drug concentrations were $5 \mu \mathrm{M}$ and the final DMSO concentration was $0.5 \%$. The negative control consisted of yeasts in $0.5 \%$ DMSO only and the positive control consisted of $0.3125 \mu \mathrm{g} / \mathrm{mL}$ amphotericin B (Sigma-Aldrich, St. Louis, MO, USA). Each library compound and control was tested in duplicate. Cultures were maintained in HMM-Ura at $37^{\circ} \mathrm{C}$ and $5 \% \mathrm{CO}_{2}$ with agitation twice daily. Growth was measured at 0 days and 4 days by measuring optical density at $595 \mathrm{~nm}\left(\mathrm{~A}_{595}\right)$ using an EL800 plate reader (BioTek Instruments, Winooski, VT, USA). Percent inhibition was calculated relative to cells treated with DMSO vehicle using the following formula: $\left[1-\left(\Delta \mathrm{OD}_{\text {Test }} / \Delta \mathrm{OD}_{\mathrm{DMSO}}\right)\right] \times 100$, where $\Delta \mathrm{OD}_{\text {Test }}=\mathrm{OD}_{595}$ at day 4 minus $\mathrm{OD}_{595}$ at day zero. For the DMSO control, $\Delta \mathrm{OD}_{\text {Test }}=\Delta \mathrm{OD}_{\mathrm{DMSO}}$, setting the $\%$ inhibition at $0 \%$.

\subsection{MIC Determination}

The MIC of top hits identified in the library screens was determined for H. capsulatum WU15 yeast cells using the methods described previously [37]. WU15 yeasts were prepared as for library screening and combined with serial 2-fold dilutions of each compound in 96 well microplates. Drug concentrations ranged from $0.03 \mu \mathrm{M}$ to $4.0 \mu \mathrm{M}$. Cultures were maintained at $37{ }^{\circ} \mathrm{C}$ and $5 \% \mathrm{CO}_{2}$ with agitation twice daily. Minimum concentrations that inhibited $100 \%$ growth relative to the day zero baseline were determined by visual inspection and confirmed by optical density measurement at $595 \mathrm{~nm}$. Similar procedures were used for MIC determination using the WU8 strain, but MIC was determined using only visual inspection due to the clumpy nature of this strain relative to WU15. MICs for Candida albicans SC5314, Saccharomyces cerevisiae, and Cryptococcus neoformans H99 were determined as described previously [67]. $\mathrm{IC}_{50}$ values were calculated using non-linear regression with GraphPad Prism for macOS, Version 8.4.3, San Diego, CA, USA.

\section{4. $\log P$ Determination}

$\log \mathrm{P}$ values of inhibitory compounds were calculated using XLogP3, Version3.0. (PubChem release 2021.05.07): http:/ / www.sioc-ccbg.ac.cn/skins/ccbgwebsite/software/ xlogp3/ (accessed on 26 August 2019) [68].

\subsection{Fungistatic/Fungicidal Testing}

To monitor the mechanism by which Sorafenib and SC -1 inhibit $H$. capsulatum growth, cultures were set up using methods identical to MIC determination, using drug concentrations at the MIC or twice the MIC values. For comparison, cells were combined with amphotericin B or fluconazole. Cultures were incubated at $37{ }^{\circ} \mathrm{C}$ and $5 \% \mathrm{CO}_{2}$. At two hours and $48 \mathrm{~h}$ following addition of compounds, samples were processed by performing serial dilutions in HMM/ uracil liquid media. Three microliters of each dilution was spotted onto HMM agarose plates supplemented with $400 \mu \mathrm{g} / \mathrm{mL}$ uracil. Plates were allowed to incubate at $37^{\circ} \mathrm{C}$ and $5 \% \mathrm{CO}_{2}$ for six days, at which point colonies are visible. Four independent experiments were performed. Fungicidal mode of action was identified as a two-log reduction in colonies at the $48 \mathrm{~h}$ time point relative to the $2 \mathrm{~h}$ time point. 


\subsection{Macrophage Lysis Assay}

Bone marrow-derived macrophages (BMDM) from 8 week-old female C57/Bl6 mice (a gift from Hooke Laboratories, Lawrence, MA, USA) were isolated from marrow differentiated in the presence of macrophage colony stimulating factor (CSF-1) as previously described [66]. Macrophage infections and lactate dehydrogenase (LDH) release assays were carried out using BMDM media [Dulbecco's modified eagle medium (DMEM) without phenol red (Gibco-ThermoFisher, Waltham, MA, USA) supplemented with $20 \%$ fetal bovine serum (Cytiva HyClone, Marlborough, MA, USA) $2 \mathrm{mM}$ glutamine, $1 \mathrm{mM} \mathrm{Na}-$ pyruvate, $100 \mathrm{U} / \mathrm{mL}$ penicillin, $100 \mu \mathrm{g} / \mathrm{mL}$ streptomycin, $400 \mu \mathrm{g} / \mathrm{mL}$ uracil and $20 \mathrm{ng} / \mathrm{mL}$ M-CSF (Gibco-ThermoFisher, Waltham, MA, USA). Phenol red was omitted from the media due to interference with the detection wavelength used for the LDH release assay. BMDM were seeded at a density of $2 \times 10^{5}$ cells/well in 24-well tissue culture treated dishes (Corning- CoStar, Cambridge, MA, USA) and allowed to adhere for two hours. Separately, log phase H. capsulatum WU15 yeast cultures were pelleted, resuspended in DMEM without phenol red and counted by hemacytometer. Yeasts were added to macrophage wells at a multiplicity of infection (MOI) of 5 . After a $2 \mathrm{~h}$ incubation period to allow for yeasts to be phagocytosed, the media was removed and macrophage monolayers were washed twice with DMEM without phenol red. Following macrophage washing, $750 \mu \mathrm{L}$ BMDM media was added to each well. The infected macrophages were incubated at $37^{\circ} \mathrm{C}$ with $5 \% \mathrm{CO}_{2}$. At $24 \mathrm{~h}$ post-infection, $250 \mu \mathrm{L}$ fresh media was added to each well. At $48 \mathrm{~h}$ following infection, LDH levels in BMDM supernatants were measured using the CytoTox Non-Radioactive cytotoxicity kit (Promega, Madison, WI, USA) as previously described $[44,66]$. The \% lysis at each time point was calculated relative to LDH levels measured in uninfected BMDM artificially induced to lyse using $1 \%$ Triton $X-100$. Three independent experiments were performed.

Supplementary Materials: The following are available online at https://www.mdpi.com/article/10 .3390 /antibiotics10101223/s1, File: Sorafenib and SC-1 do not act synergistically with fluconazole or amphotericin B.

Author Contributions: Conceptualization, C.B. and J.F.; Data curation, C.B. and J.F.; Formal analysis, C.B., J.F., M.L. and K.B.; Funding acquisition, C.B. and J.F.; Investigation, M.L., K.B., J.M., D.L. and A.C.; Methodology, C.B. and J.F.; Project administration, C.B.; Resources, C.B. and J.F.; Supervision, C.B.; Validation, C.B. and M.L.; Visualization, C.B.; Writing-original draft, C.B.; Writing-review and editing, C.B. and J.F. All authors have read and agreed to the published version of the manuscript.

Funding: This research was funded by the Laurence M. Demers Professorship in the Life Sciences, Merrimack College (C.B.) and by Merrimack College Faculty Development Grants (C.B. and J.F.). This study received no external funding.

Data Availability Statement: Data is contained within the article and supplementary material.

Acknowledgments: We are indebted to the University of North Carolina Structural Genomics Consortium for providing the GlaxoSmithKline Published Kinase Inhibitor Set (PKIS) under an open access Material Transfer and Trust Agreement. Bone marrow derived macrophages were generated using femurs provided as a gift by Hooke Laboratories, Lawrence, MA. We would like to thank Violet Tran for media preparation and Lauri Gibbons for administrative and laboratory support.

Conflicts of Interest: The authors declare no conflict of interest. The funders had no role in the design of the study; in the collection, analyses, or interpretation of data; in the writing of the manuscript, or in the decision to publish the results.

\section{References}

1. Pfaller, M.A.; Diekema, D. Epidemiology of Invasive Mycoses in North America. Crit. Rev. Microbiol. 2010, 36, 1-53. [CrossRef] [PubMed]

2. Benedict, K.; Jackson, B.R.; Chiller, T.; Beer, K.D. Estimation of Direct Healthcare Costs of Fungal Diseases in the United States. Clin. Infect. Dis. 2019, 68, 1791-1797. [CrossRef] [PubMed]

3. Horwath, M.C.; Fecher, R.A.; Deepe, G.S. Histoplasma capsulatum, lung infection and immunity. Futur. Microbiol. 2015, 10, 967-975. [CrossRef] [PubMed] 
4. Jain, V.V.; Evans, T.; Peterson, M.W. Reactivation histoplasmosis after treatment with anti-tumor necrosis factor $\alpha$ in a patient from a nonendemic area. Respir. Med. 2006, 100, 1291-1293. [CrossRef] [PubMed]

5. Vergidis, P.; Avery, R.K.; Wheat, L.J.; Dotson, J.L.; Assi, M.A.; Antoun, S.A.; Hamoud, K.A.; Burdette, S.D.; Freifeld, A.G.; McKinsey, D.S.; et al. Histoplasmosis Complicating Tumor Necrosis Factor- $\alpha$ Blocker Therapy: A Retrospective Analysis of 98 Cases. Clin. Infect. Dis. 2015, 61, 409-417. [CrossRef] [PubMed]

6. Nakelchik, M.; Mangino, J.E. Reactivation of histoplasmosis after treatment with infliximab. Am. J. Med. 2002, 112, 78-79. [CrossRef]

7. Lucey, O.; Carroll, I.; Bjorn, T.; Millar, M. Reactivation of latent Histoplasma and disseminated cytomegalovirus in a returning traveller with ulcerative colitis. JMM Case Rep. 2018, 5, e005170. [CrossRef]

8. Perfect, J.R. The antifungal pipeline: A reality check. Nat. Rev. Drug Discov. 2017, 16, 603-616. [CrossRef]

9. Odds, F.C.; Brown, A.J.; Gow, N.A. Antifungal agents: Mechanisms of action. Trends Microbiol. 2003, 11, 272-279. [CrossRef]

10. Wheat, L.J.; Freifeld, A.G.; Kleiman, M.B.; Baddley, J.W.; McKinsey, D.S.; Loyd, J.; Kauffman, C.A. Clinical Practice Guidelines for the Management of Patients with Histoplasmosis: 2007 Update by the Infectious Diseases Society of America. Clin. Infect. Dis. 2007, 45, 807-825. [CrossRef]

11. Nett, J.E.; Andes, D.R. Antifungal Agents: Spectrum of Activity, Pharmacology, and Clinical Indications. Infect. Dis. Clin. North Am. 2016, 30, 51-83. [CrossRef] [PubMed]

12. Bates, D.W.; Su, L.; Yu, D.T.; Chertow, G.M.; Seger, D.L.; Gomes, D.R.J.; Dasbach, E.J.; Platt, R. Mortality and Costs of Acute Renal Failure Associated with Amphotericin B Therapy. Clin. Infect. Dis. 2001, 32, 686-693. [CrossRef]

13. Pappas, P.G.; Kauffman, C.A.; Andes, D.; Clancy, C.J.; Marr, K.A.; Ostrosky-Zeichner, L.; Reboli, A.C.; Schuster, M.G.; Vazquez, J.A.; Walsh, T.J.; et al. Clinical Practice Guideline for the Management of Candidiasis: 2016 Update by the Infectious Diseases Society of America. Clin. Infect. Dis. 2016, 62, e1-e50. [CrossRef]

14. Nakai, T.; Uno, J.; Ikeda, F.; Tawara, S.; Nishimura, K.; Miyaji, M. In Vitro Antifungal Activity of Micafungin (FK463) against Dimorphic Fungi: Comparison of Yeast-Like and Mycelial Forms. Antimicrob. Agents Chemother. 2003, 47, 1376-1381. [CrossRef]

15. Hage, C.A.; Connolly, P.; Horan, D.; Durkin, M.; Smedema, M.; Zarnowski, R.; Smith, P.; Wheat, L.J. Investigation of the Efficacy of Micafungin in the Treatment of Histoplasmosis Using Two North American Strains of Histoplasma capsulatum. Antimicrob. Agents Chemother. 2011, 55, 4447-4450. [CrossRef]

16. Roemer, T.; Krysan, D.J. Antifungal Drug Development: Challenges, Unmet Clinical Needs, and New Approaches. Cold Spring Harb. Perspect. Med. 2014, 4, a019703. [CrossRef]

17. Pushpakom, S.; Iorio, F.; Eyers, P.A.; Escott, K.J.; Hopper, S.; Wells, A.; Doig, A.; Guilliams, T.; Latimer, J.; McNamee, C.; et al. Drug repurposing: Progress, challenges and recommendations. Nat. Rev. Drug Discov. 2019, 18, 41-58. [CrossRef]

18. Bamborough, P. System-based drug discovery within the human kinome. Expert Opin. Drug Discov. 2012, 7, 1-18. [CrossRef]

19. Wu, P.; Nielsen, T.E.; Clausen, M.H. FDA-approved small-molecule kinase inhibitors. Trends Pharmacol. Sci. 2015, 36, 422-439. [CrossRef]

20. Edwards, A.M.; Bountra, C.; Kerr, D.J.; Willson, T.M. Open access chemical and clinical probes to support drug discovery. Nat. Chem. Biol. 2009, 5, 436-440. [CrossRef]

21. Hohmann, S. Osmotic Stress Signaling and Osmoadaptation in Yeasts. Microbiol. Mol. Biol. Rev. 2002, 66, 300-372. [CrossRef]

22. Andrews, P.; Stark, M. Type 1 protein phosphatase is required for maintenance of cell wall integrity, morphogenesis and cell cycle progression in Saccharomyces cerevisiae. J. Cell Sci. 2000, 113, 507-520. [CrossRef] [PubMed]

23. Gustin, M.C.; Albertyn, J.; Alexander, M.; Davenport, K. MAP Kinase Pathways in the Yeast Saccharomyces cerevisiae. Microbiol. Mol. Biol. Rev. 1998, 62, 1264-1300. [CrossRef]

24. Posas, F. Osmotic Activation of the HOG MAPK Pathway via Ste11p MAPKKK: Scaffold Role of Pbs2p MAPKK. Science 1997, 276, 1702-1705. [CrossRef] [PubMed]

25. Wojda, I.; Monge, R.A.; Bebelman, J.-P.; Mager, W.H.; Siderius, M. Response to high osmotic conditions and elevated temperature in Saccharomyces cerevisiae is controlled by intracellular glycerol and involves coordinate activity of MAP kinase pathways. Microbiology 2003, 149, 1193-1204. [CrossRef] [PubMed]

26. Leberer, E.; Harcus, D.; Dignard, D.; Johnson, L.; Ushinsky, S.; Thomas, D.; Schröppel, K. Ras links cellular morphogenesis to virulence by regulation of the MAP kinase and cAMP signalling pathways in the pathogenic fungus Candida albicans. Mol. Microbiol. 2008, 42, 673-687. [CrossRef] [PubMed]

27. Csank, C.; Makris, C.; Meloche, S.; Schröppel, K.; Röllinghoff, M.; Dignard, D.; Thomas, D.; Whiteway, M. Derepressed Hyphal Growth and Reduced Virulence in a VH1 Family-related Protein Phosphatase Mutant of the Human Pathogen Candida albicans. Mol. Biol. Cell 1997, 8, 2539-2551. [CrossRef] [PubMed]

28. Li, X.; Du, W.; Zhao, J.; Zhang, L.; Zhu, Z.; Jiang, L. The MAP kinase-activated protein kinase Rck2p regulates cellular responses to cell wall stresses, filamentation and virulence in the human fungal pathogen Candida albicans. FEMS Yeast Res. 2010, 10, 441-451. [CrossRef] [PubMed]

29. Lee, K.-T.; So, Y.-S.; Yang, D.-H.; Jung, K.-W.; Choi, J.; Lee, D.-G.; Kwon, H.; Jang, J.; Wang, L.L.; Cha, S.; et al. Systematic functional analysis of kinases in the fungal pathogen Cryptococcus neoformans. Nat. Commun. 2016, 7, 12766. [CrossRef]

30. Bahn, Y.-S.; Kojima, K.; Cox, G.M.; Heitman, J. Specialization of the HOG Pathway and Its Impact on Differentiation and Virulence of Cryptococcus neoformans. Mol. Biol. Cell 2005, 16, 2285-2300. [CrossRef] 
31. Kim, S.-Y.; Ko, Y.-J.; Jung, K.-W.; Strain, A.; Nielsen, K.; Bahn, Y.-S. Hrk1 Plays Both Hog1-Dependent and -Independent Roles in Controlling Stress Response and Antifungal Drug Resistance in Cryptococcus neoformans. PLoS ONE 2011, 6, e18769. [CrossRef]

32. Manning, G.; Plowman, G.D.; Hunter, T.; Sudarsanam, S. Evolution of protein kinase signaling from yeast to man. Trends Biochem. Sci. 2002, 27, 514-520. [CrossRef]

33. Kojima, K.; Bahn, Y.-S.; Heitman, J. Calcineurin, Mpk1 and Hog1 MAPK pathways independently control fludioxonil antifungal sensitivity in Cryptococcus neoformans. Microbiology 2006, 152, 591-604. [CrossRef]

34. Elkins, J.M.; Fedele, V.; Szklarz, M.; Azeez, K.R.A.; Salah, E.; Mikolajczyk, J.; Romanov, S.; Sepetov, N.; Huang, X.-P.; Roth, B.L.; et al. Comprehensive characterization of the Published Kinase Inhibitor Set. Nat. Biotechnol. 2016, 34, 95-103. [CrossRef]

35. Drewry, D.; Willson, T.M.; Zuercher, W.J. Seeding Collaborations to Advance Kinase Science with the GSK Published Kinase Inhibitor Set (PKIS). Curr. Top. Med. Chem. 2014, 14, 340-342. [CrossRef]

36. Edwards, J.A.; Kemski, M.M.; Rappleye, C.A. Identification of an Aminothiazole with Antifungal Activity against Intracellular Histoplasma capsulatum. Antimicrob. Agents Chemother. 2013, 57, 4349-4359. [CrossRef] [PubMed]

37. Goughenour, K.D.; Balada-Llasat, J.-M.; Rappleye, C.A. Quantitative Microplate-Based Growth Assay for Determination of Antifungal Susceptibility of Histoplasma capsulatum Yeasts. J. Clin. Microbiol. 2015, 53, 3286-3295. [CrossRef] [PubMed]

38. Silverman, R.B.; Holladay, M.W. The Organic Chemistry of Drug Design and Drug Action. In The Organic Chemistry of Drug Design and Drug Action, 3rd ed.; Academic Press: Cambridge, MA, USA, 2014.

39. Lipinski, C.A.; Lombardo, F.; Dominy, B.W.; Feeney, P.J. Experimental and computational approaches to estimate solubility and permeability in drug discovery and development settings. Adv. Drug Deliv. Rev. 2012, 64, 4-17. [CrossRef]

40. Ghose, A.K.; Viswanadhan, V.N.; Wendoloski, J.J. A Knowledge-Based Approach in Designing Combinatorial or Medicinal Chemistry Libraries for Drug Discovery. 1. A Qualitative and Quantitative Characterization of Known Drug Databases. J. Comb. Chem. 1999, 1, 55-68. [CrossRef] [PubMed]

41. Tai, W.-T.; Cheng, A.-L.; Shiau, C.-W.; Huang, H.-P.; Huang, J.-W.; Chen, P.-J.; Chen, K.-F. Signal transducer and activator of transcription 3 is a major kinase-independent target of sorafenib in hepatocellular carcinoma. J. Hepatol. 2011, 55, 1041-1048. [CrossRef]

42. Chiu, C.-M.; Huang, S.-Y.; Chang, S.-F.; Liao, K.-F.; Chiu, S.-C. Synergistic antitumor effects of tanshinone IIA and sorafenib or its derivative SC-1 in hepatocellular carcinoma cells. OncoTargets Ther. 2018, 11, 1777-1785. [CrossRef] [PubMed]

43. Su, T.-H.; Shiau, C.-W.; Jao, P.; Liu, C.-H.; Liu, C.-J.; Tai, W.-T.; Jeng, Y.-M.; Yang, H.-C.; Tseng, T.-C.; Huang, H.-P.; et al. Sorafenib and its derivative SC-1 exhibit antifibrotic effects through signal transducer and activator of transcription 3 inhibition. Proc. Natl. Acad. Sci. USA 2015, 112, 7243-7248. [CrossRef] [PubMed]

44. Isaac, D.T.; Berkes, C.A.; English, B.C.; Murray, D.H.; Lee, Y.N.; Coady, A.; Sil, A. Macrophage cell death and transcriptional response are actively triggered by the fungal virulence factor $\mathrm{Cbp} 1$ during H.capsulatum infection. Mol. Microbiol. 2015, 98, 910-929. [CrossRef] [PubMed]

45. Patel, G.; Roncal, N.E.; Lee, P.J.; Leed, S.E.; Erath, J.; Rodriguez, A.; Sciotti, R.; Pollastri, M.P. Repurposing human Aurora kinase inhibitors as leads for anti-protozoan drug discovery. MedChemComm 2014, 5, 655-658. [CrossRef] [PubMed]

46. Dichiara, M.; Marrazzo, A.; Prezzavento, O.; Collina, S.; Rescifina, A.; Amata, E. Repurposing of Human Kinase Inhibitors in Neglected Protozoan Diseases. ChemMedChem 2017, 12, 1235-1253. [CrossRef]

47. Stylianou, M.; Kulesskiy, E.; Lopes, J.P.; Granlund, M.; Wennerberg, K.; Urban, C.F. Antifungal Application of Nonantifungal Drugs. Antimicrob. Agents Chemother. 2013, 58, 1055-1062. [CrossRef]

48. Butts, A.; DiDone, L.; Koselny, K.; Baxter, B.K.; Chabrier-Rosello, Y.; Wellington, M.; Krysan, D.J. A Repurposing Approach Identifies Off-Patent Drugs with Fungicidal Cryptococcal Activity, a Common Structural Chemotype, and Pharmacological Properties Relevant to the Treatment of Cryptococcosis. Eukaryot. Cell 2012, 12, 278-287. [CrossRef]

49. Khalil, A.; Edwards, J.A.; Rappleye, C.A.; Tjarks, W. Design, synthesis, and biological evaluation of aminothiazole derivatives against the fungal pathogens Histoplasma capsulatum and Cryptococcus neoformans. Bioorganic Med. Chem. 2015, $23,532-547$. [CrossRef]

50. Catalano, A.; Iacopetta, D.; Sinicropi, M.S.; Franchini, C. Diarylureas as Antitumor Agents. Appl. Sci. 2021, 11, 374. [CrossRef]

51. Garuti, L.; Roberti, M.; Bottegoni, G.; Ferraro, M. Diaryl Urea: A Privileged Structure in Anticancer Agents. Curr. Med. Chem. 2016, 23, 1528-1548. [CrossRef]

52. Catalano, A.; Iacopetta, D.; Pellegrino, M.; Aquaro, S.; Franchini, C.; Sinicropi, M. Diarylureas: Repositioning from Antitumor to Antimicrobials or Multi-Target Agents against New Pandemics. Antibiotics 2021, 10, 92. [CrossRef]

53. Iacopetta, D.; Catalano, A.; Ceramella, J.; Saturnino, C.; Salvagno, L.; Ielo, I.; Drommi, D.; Scali, E.; Plutino, M.; Rosace, G.; et al. The Different Facets of Triclocarban: A Review. Molecules 2021, 26, 2811. [CrossRef] [PubMed]

54. Catalano, A.; Rosato, A.; Salvagno, L.; Iacopetta, D.; Ceramella, J.; Fracchiolla, G.; Sinicropi, M.; Franchini, C. BenzothiazoleContaining Analogues of Triclocarban with Potent Antibacterial Activity. Antibiotics 2021, 10, 803. [CrossRef] [PubMed]

55. Lundberg, L.; Brahms, A.; Hooper, I.; Carey, B.D.; Lin, S.-C.; Dahal, B.; Narayanan, A.; Kehn-Hall, K. Repurposed FDA-Approved drug sorafenib reduces replication of Venezuelan equine encephalitis virus and other alphaviruses. Antivir. Res. 2018, $157,57-67$. [CrossRef] 
56. Kocyigit-Kaymakcioglu, B.; Celen, A.O.; Tabanca, N.; Ali, A.; Khan, S.I.; Khan, I.A.; Wedge, D.E. Synthesis and Biological Activity of Substituted Urea and Thiourea Derivatives Containing 1,2,4-Triazole Moieties. Molecules 2013, 18, 3562-3576. [CrossRef] [PubMed]

57. Del Poeta, M.; Chen, S.-F.; Von Hoff, D.; Dykstra, C.C.; Wani, M.C.; Manikumar, G.; Heitman, J.; Wall, M.E.; Perfect, J.R Comparison of In Vitro Activities of Camptothecin and Nitidine Derivatives against Fungal and Cancer Cells. Antimicrob. Agents Chemother. 1999, 43, 2862-2868. [CrossRef]

58. Heitman, J.; Movva, N.; Hall, M. Targets for cell cycle arrest by the immunosuppressant rapamycin in yeast. Science 1991, 253, 905-909. [CrossRef]

59. Cutler, N.S.; Heitman, J.; Cardenas, M.E. STT4 Is an Essential Phosphatidylinositol 4-Kinase That Is a Target of Wortmannin in Saccharomyces cerevisiae. J. Biol. Chem. 1997, 272, 27671-27677. [CrossRef] [PubMed]

60. Liu, L.; Cao, Y.; Chen, C.; Zhang, X.; McNabola, A.; Wilkie, D.; Wilhelm, S.; Lynch, M.; Carter, C. Sorafenib Blocks the RAF/MEK/ERK Pathway, Inhibits Tumor Angiogenesis, and Induces Tumor Cell Apoptosis in Hepatocellular Carcinoma Model PLC/PRF/5. Cancer Res. 2006, 66, 11851-11858. [CrossRef] [PubMed]

61. Coriat, R.; Nicco, C.; Chéreau, C.; Mir, O.; Alexandre, J.; Ropert, S.; Weill, B.; Chaussade, S.; Goldwasser, F.; Batteux, F. SorafenibInduced Hepatocellular Carcinoma Cell Death Depends on Reactive Oxygen Species Production In Vitro and In Vivo. Mol. Cancer Ther. 2012, 11, 2284-2293. [CrossRef]

62. Liu, C.-Y.; Tseng, L.-M.; Su, J.-C.; Chang, K.-C.; Chu, P.-Y.; Tai, W.-T.; Shiau, C.-W.; Chen, K.-F. Novel sorafenib analogues induce apoptosis through SHP-1 dependent STAT3 inactivation in human breast cancer cells. Breast Cancer Res. 2013, 15, R63. [CrossRef]

63. Zhao, X.; Mehrabi, R.; Xu, J.-R. Mitogen-Activated Protein Kinase Pathways and Fungal Pathogenesis. Eukaryot. Cell 2007, 6, 1701-1714. [CrossRef]

64. Knauth, P.; Reichenbach, H. On the Mechanism of Action of the Myxobacterial Fungicide Ambruticin. J. Antibiot. 2000, 53, 1182-1190. [CrossRef] [PubMed]

65. Marion, C.L.; Rappleye, C.; Engle, J.T.; Goldman, W.E. An $\alpha-(1,4)$-amylase is essential for $\alpha-(1,3)$-glucan production and virulence in Histoplasma capsulatum. Mol. Microbiol. 2006, 62, 970-983. [CrossRef] [PubMed]

66. Isaac, D.T.; Coady, A.; Van Prooyen, N.; Sil, A. The 3-Hydroxy-Methylglutaryl Coenzyme a Lyase HCL1 Is Required for Macrophage Colonization by Human Fungal Pathogen Histoplasma capsulatum. Infect. Immun. 2013, 81, 411-420. [CrossRef] [PubMed]

67. Rex, J.H.; Alexander, B.D.; Andes, D.; Arthington-Skaggs, B.; Brown, S.D.; Chaturvedi, V.; Ghannoum, M.A.; Espinel-Ingroff, A.; Knapp, C.C.; Ostrosky-Zeichner, L.; et al. Reference Method for Broth Dilution Antifungal Susceptibility Testing of Yeasts: Approved Standard, 3rd ed.; Clinical and Laboratory Standards Institute: Wayne, PA, USA, 2008.

68. Cheng, T.; Zhao, Y.; Li, X.; Lin, F.; Xu, Y.; Zhang, X.; Li, A.Y.; Wang, R.; Lai, L. Computation of Octanol-Water Partition Coefficients by Guiding an Additive Model with Knowledge. J. Chem. Inf. Model. 2007, 47, 2140-2148. [CrossRef] 